\title{
A method to determine the onset voltage of single and arrays of electrospray emitters
}

\author{
Renato Krpoun a) and Herbert R. Shea \\ Ecole Polytechnique Fédérale de Lausanne, 1015 Lausanne, Switzerland
}

(Received 13 April 2008; accepted 24 July 2008; published online 25 September 2008)

\begin{abstract}
This paper reports on an accurate and rapid method to compute the onset voltage of a single or an array of electrospray emitters with complex geometries and on the correlation of the simulation with experimental data. This method permits the exact determination of the onset voltage based only on the surface tension of the sprayed liquid and on the emitter geometry. The approach starts by determining the voltage at which electrostatic forces and surface tension forces are equal for a sharpening conic surface at the tip of a capillary as a function of the apex radius of the liquid. By tracing the curve of this computed equilibrium voltage as a function of the apex radius, the onset voltage for a liquid surface with the Taylor half-angle of $49.3^{\circ}$ or larger can be determined. For smaller cone half-angles the method is only applicable to ionic sprays as an approximate knowledge of the critical field for ion emission is necessary. The combination of analytical models and finite element tools used to compute the necessary parameters is described. The method is validated on a complex microelectromechanical system emitter geometry as well as on a linear array of electrospray emitters. Finally an empirical model of the behavior of the electric field near the apex of a conic surface with asymptotes at a fixed half-angle is proposed, which allows establishing a simple method for onset voltage determination. (C) 2008 American Institute of Physics.
\end{abstract}

[DOI: $10.1063 / 1.2981077$ ]

\section{INTRODUCTION}

A conductive liquid at the tip of a wetted needle or a filled capillary will, as the voltage is increased, deform into a cone. Once a threshold voltage, called the "onset voltage," is reached the electric stress at the apex of the liquid surface overcomes surface tension and a spray of particles is ejected toward a counterelectrode. These sources of charged particles known as electrosprays (ES) are commonly used in ES ionization for mass spectrometry of macromolecules (see Fig. 1).

A model predicting the onset voltage is helpful in the development of any type of ES or liquid metal ion source, and becomes a powerful tool when designing microelectromechanical system (MEMS) emitter arrays for printing applications, for ES mass spectrometry, or for space propulsion. In particular, electric field distortion of adjacent emitters in symmetrical arrays leads to inhomogeneities in onset voltages across the array and should be minimized. The feasibility and operability of high density MEMS emitter arrays with individual extractor electrodes have been demonstrated for internally ${ }^{1-4}$ and externally ${ }^{5,6}$ fed emitters. An example of microfabricated arrays is shown in Fig. 2.

The most common model to determine the onset voltage assumes a paraboloid or hyperboloid tip facing an extraction electrode. ${ }^{7,8}$ It assumes that once the electrical stress at the tip of the needle exceeds the surface tension of the liquid a cone will form and emission will start. This approach has shown good agreement with experiments using long slender needles ${ }^{7}$ but does not produce satisfactory results for lower

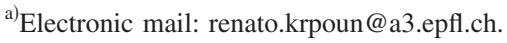

aspect ratio structures as can be made for MEMS. One difficulty is the complex geometry surrounding the emitter and distorting the electric field around the liquid surface. Pioneering work on the effect of capillary spacing in arrays has been done by Regele et al. ${ }^{9}$ and Quang Tran Si et al. ${ }^{10}$ Both authors studied the influence of array pitch on the onset voltage and present an analytical model to calculate the onset voltage in their arrays which requires the experimental determination of a calibration factor.

The objective of this paper is to present a method to quantitatively determine the onset voltage of a single or arrays of ES taking into account the real emitter-extractor geometry and the surface tension of the liquid. The method is based on an analytical approach but requires a finite element model (FEM) to solve the Laplace equation. Our approach assumes a quasistatic evolution of the liquid at the tip of the capillary and does not consider fluid dynamics or space charge effects. As will be shown in Sec. III A the electric field at the apex of a liquid meniscus evolving within the

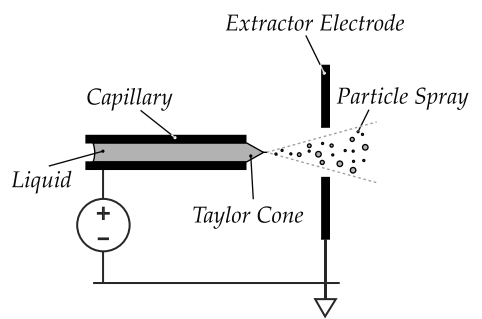

FIG. 1. Principle of operation of an ES: a voltage difference is applied between a conductive liquid in a capillary and an extractor electrode. Once the onset voltage is reached the liquid deforms into a cone and charged particles are emitted. 


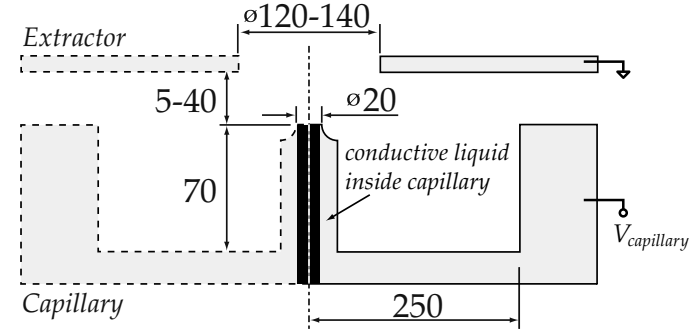

(a) Geometry

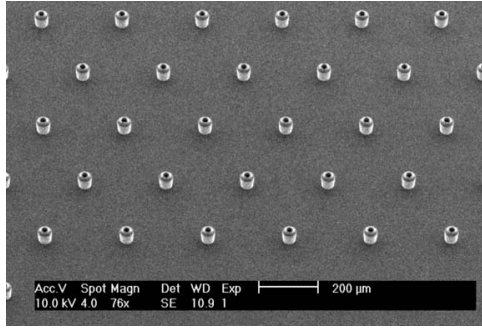

(b) Capillary Array

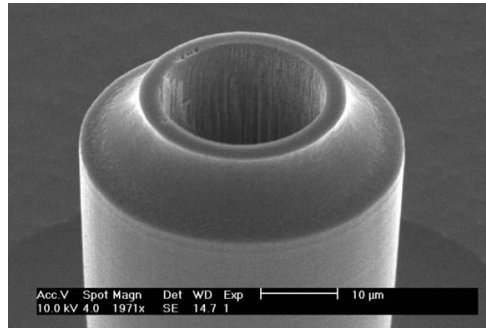

(c) Capillary Tip

FIG. 2. Microfabricated arrays (Ref. 3): (a) is a schematic cross section of the emitter geometry consisting of a capillary emitter and an extractor electrode. The dimensions in the illustration are in microns. (b) and (c) are SEM photographs of an array of capillaries and the detail of a tip prior to extractor electrode integration.

Taylor cone half-angle of $49.3^{\circ}$ is inversely proportional to the square root of the apex radius. This behavior allows predicting the onset voltage for purely ionic mode and droplet mode jets without knowing the electric field level necessary to initiate emission. Onset voltage modeling for other cone half-angles will be discussed in Sec. II C. We will show that for angles below $49.3^{\circ}$ the knowledge of a "threshold" electric field at the apex of the liquid is necessary to determine at which voltage emission starts.

Finally we limit our study to capillaries with flat tops, but the same approach could be taken for determining onset voltages on the tip of needles. So far, only problems with an axisymmetric liquid cone have been investigated.

\section{METHODOLOGY AND THEORETICAL BACKGROUND}

The method presented below is a four step process: (a) define a geometry consisting of a fixed capillary on top of which a conductive liquid is placed with a predetermined shape, this shape is varied from flat to a cone and can be expressed by the radius of curvature $r_{a}$ at the apex; (b) compute the relationship between the electric field at the apex and the applied voltage for different apex radii of curvature (it is assumed the liquid and capillary are perfect conductors); (c) determine the equilibrium voltage at which the electrostatic and surface tension forces are equal for any $r_{a}$; and (d) using the plot of equilibrium voltage $V_{0}$ versus $r_{a}$, and the approximate knowledge of the electric field required for ion emission, determine with high accuracy the onset voltage for ES.

Because this model only considers voltages up to emission, no emission occurs; hence, there is no space charge and so the combination of the static geometry of the emitter with the evolving surface defines the boundary conditions of the Laplace problem. Figure 3 shows a series of photographs taken of tip sharpening. Figures 3(a) and 3(b) represent steady states below the onset voltage. Once a threshold voltage is reached the liquid drop snaps over into a Taylor cone [Fig. 3(c)]. Because we assume a quasistatic case, the liquid surface and capillary structure can be considered at an equipotential. Once snap-over occurs charge relaxation becomes an issue for low conductivity liquids. The momentum gained by the liquid needs to be dissipated or can lead to oscillations of the liquid surface. For our model we will only consider the point up to snap-over for sprays operating in droplet mode, thus ignoring all dynamic aspects, such as jet formation. For ionic mode emissions a damped deformation into a cone (without a jet) during snap-over will be assumed up to the point where the critical field for ion emission is reached at the apex.

This chapter is organized in the same order as the method's practical application. The model requires the superposition of a liquid surface on top of a static capillary geometry; therefore, at first the variable liquid surface is constructed using a function closely reproducing the liquids behavior (Sec. II A). This surface is then combined with the static emitter geometry, and the Laplace equation is solved for an arbitrary potential (Sec. II B). Due to the linear nature of the problem the relationship between electric field and potential can be extrapolated to solve the equilibrium equation relating electric stress and the surface tension (Sec. II C). The interpretation of the obtained equilibrium voltage curves leads to the determination of the onset voltage.

\section{A. Liquid surface modeling}

For ES the shape of the liquid surface in transition from a flat surface into a cone is only poorly documented and varies depending on the type of liquid, the applied voltages, and the flow regime. Observations by Driesel et al. ${ }^{11}$ of liquid metal ion sources show a stable cone for very low emission currents and a cone half-angle close to $49.3^{\circ}$ as predicted by Taylor. ${ }^{12}$ Our observations (Fig. 3) of the liquid on the tip of a $360 \mu \mathrm{m}$ capillary show an initial sharpening of the liquid surface as the voltage is increased, and once a threshold voltage is reached, the surface deforms suddenly into a cone.
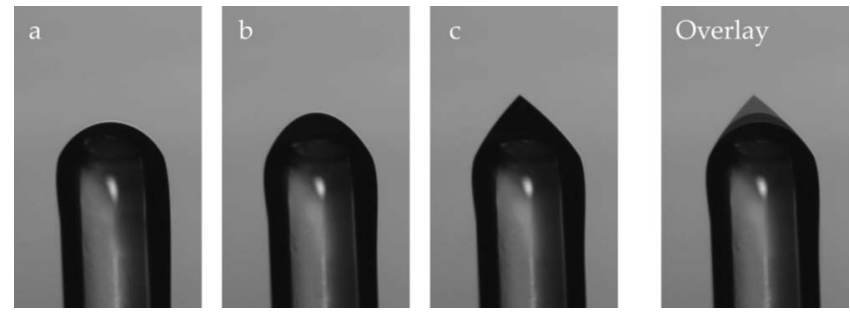

FIG. 3. Sharpening of the liquid surface at the tip of a $360 \mu \mathrm{m}$ outer diameter capillary. Photographs were taken at ambient pressure while spray-

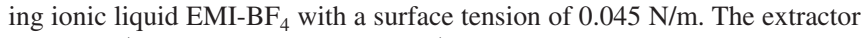
electrode (not visible in these images) is a plate placed a few millimeters above the capillary. 


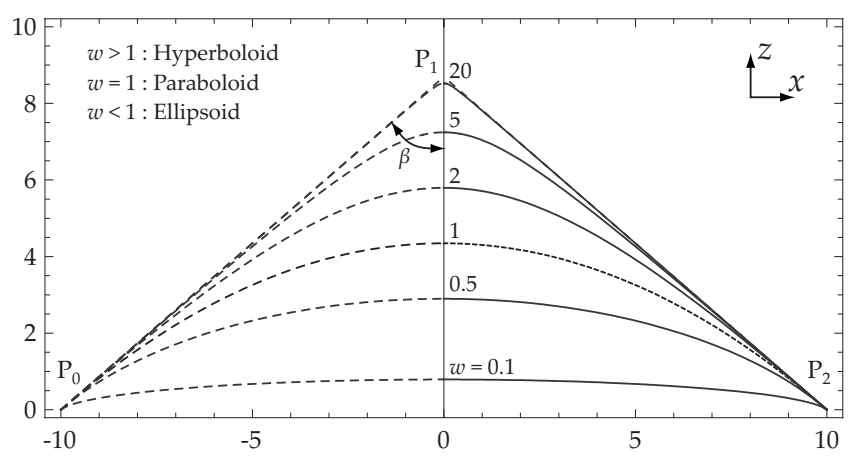

FIG. 4. Plot of the conic function used to model the formation of a Taylor cone with $p_{x}=10$ and $\beta=49.3^{\circ}$.

Near the onset voltage the emitted spray may operate in a pulsating mode rather than in a steady state. A slight increase in the flow rate or voltage leads to a constant current operating mode. A very large increase in voltage will lead to unstable operation and multiple emission sites per capillary. Two research groups have studied pulsating ES with high speed cameras ${ }^{13,14}$ and have defined four distinct modes of operation with a cycle duration around $500 \mu$ s. During phase I the liquid accumulates at the tip of the capillary and is close to spherical shape. In phase II the Maxwell stress transforms the surface into a cone. Once it transitions into phase III the cone deforms into a cusplike structure with a jet; finally, in phase IV the liquid retreats into a drop and the cycle restarts.

Based on observations made in our experiment and on the shapes observed in pulsating ES we chose to model the sharpening of the apex by a conic section. The chosen function corresponds to a straight line for an infinite radius of curvature, $r_{a}=\infty$, and a perfect cone for zero apex radius, $r_{a}=0$. An arc of a conic having such properties can be expressed mathematically as a Bernstein-Bézier curve ${ }^{15}$

$$
\vec{C}(t)=\frac{B_{0,2}(t) \vec{P}_{0}+B_{1,2}(t) w \vec{P}_{1}+B_{2,2}(t) \vec{P}_{2}}{B_{0,2}(t)+B_{1,2}(t) w+B_{2,2}(t)}
$$

where $B_{i, p}(t)$ are the $p$ th degree Bernstein basis functions defined by

$$
B_{i, p}(t)=\left(\begin{array}{c}
p \\
i
\end{array}\right) t^{i}(1-t)^{p-i} \quad(i=0,1, \ldots, p),
$$

$$
\left(\begin{array}{c}
p \\
i
\end{array}\right): \text { Binomial coefficient }
$$

The parameter $w \in[0, \infty)$ defines the apex radius of curvature $r_{a}$ and $t \in[0,0.5]$ is the running parameter. Equation (1) defines a one parameter family of conics passing through $\vec{P}_{0}$ and $\vec{P}_{2}$. The conic is further tangent to the chords $\left[\vec{P}_{0}, \vec{P}_{1}\right]$ and $\left[\vec{P}_{1}, \vec{P}_{2}\right]$. Figure 4 plots the function, bounded by the asymptotes of the Taylor half-angle $\beta$ for different apex radii. For an arc having its apex on the $z=0$ line and bound by the cone half-angle $\beta$, the chords can be written as

$$
\vec{P}_{0}=-\vec{P}_{2}=\left(\begin{array}{c}
-p_{x} \\
0
\end{array}\right), \quad \vec{P}_{1}=\left(\begin{array}{c}
0 \\
p_{x} \cot (\beta)
\end{array}\right) .
$$

The surface obtained by spinning this curve around the $z$-axis in a Cartesian coordinate system yields the following parametric surface equations:

$$
\begin{aligned}
& x(t, w, \phi)=\frac{(1-2 t) p_{x}}{1-2 t(1-t)(1-w)} \cos \phi, \\
& y(t, w, \phi)=\frac{(1-2 t) p_{x}}{1-2 t(1-t)(1-w)} \sin \phi, \\
& z(t, w, \phi)=\frac{2(1-t) t w \cot \beta p_{x}}{1-2 t(1-t)(1-w)},
\end{aligned}
$$

where $\phi \in[0,2 \pi)$. In the Cartesian representation, Eq. (4) can be written as

$$
\left(\frac{\left|1-w^{2}\right|}{w} \frac{z}{p_{x} \cot \beta}+w\right)^{2}+\left(1-w^{2}\right)\left(\frac{r}{p_{x}}\right)^{2}=1,
$$

where $r^{2}=x^{2}+y^{2}$. The radius of curvature at the apex is

$$
r_{a}=\frac{p_{x}}{w} \tan \beta .
$$

\section{B. Solving the Laplace equation}

The absence of space charge allows writing the wellknown relationships between electric potential $\Phi$ and field $E^{16}$

$$
\begin{aligned}
& \nabla^{2} \Phi(x, y, z)=0, \\
& \vec{E}=-\vec{\nabla} \Phi .
\end{aligned}
$$

The boundary conditions are the two potentials of the conductors: the extraction electrode, and the capillary and liquid, which form one conductor. The complexity of the geometry makes it difficult to find a solution using analytical methods; thus, a FEM is constructed to compute the electric field distribution based on the potential on the extractor electrode and on the capillary.

To illustrate the method, the construction of a MEMS emitter $^{3}$ will be given as example. To simplify the geometry an axisymmetrical model is created, where the adjacent capillaries or structures are represented by a solid sidewall. To decrease the computation time only a small slice of the symmetrical geometry is modeled by carefully constraining the lateral faces of the slice to have the same potential distribution (Fig. 5). To generate cyclic constraints in ANSYS, a lateral face is first meshed with surface elements; this mesh is then replicated onto the other face. The next step consists in volume-meshing the entire slice, removing the unnecessary surface elements, and placing cyclical constraints on the lateral faces to reproduce the behavior of the axisymmetric geometry. Finally surface potentials are defined, the capillary and liquid surfaces are set to an arbitrary voltage $V_{\xi}$, and the 


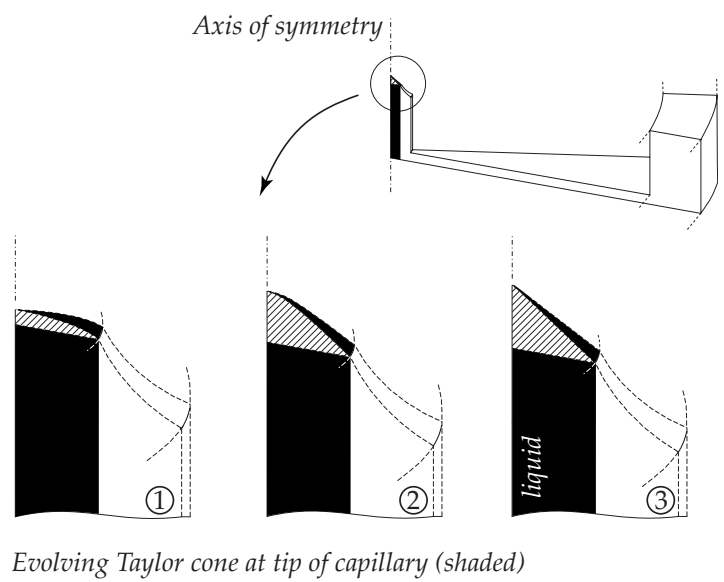

FIG. 5. Slice of the geometry used to compute the electric field distribution for three radii of curvature. The liquid and capillary walls are assumed to be perfect conductors. The extractor electrode is not shown for clarity, and is as in Fig. 2(a).

extractor electrode is placed at zero potential. At this point the solver can be launched and the resulting electric field $E_{a_{\xi}}$ at the apex of the liquid is obtained.

This procedure is repeated for a series of liquid shapes (described in Sec. II A) sharpening into a Taylor cone. For each liquid shape (i.e., for each radius of curvature $r_{a}$ ), the ratio

$$
\kappa=\frac{E_{a_{\xi}}}{V_{\xi}},
$$

can be obtained from the FEM solution, allowing the electric field $E_{a_{\xi}}$ to be computed for any applied voltage to the capillary using the expression

$$
E_{\text {apex }}=\kappa V_{\text {capillary }} \text {. }
$$

When the electric field of sharp surfaces having nanometer scale radii surrounded by macroscopic structures is computed, particular care needs to be taken when setting up the mesh. Although a proof of the validity of a finite element tool for all geometries is not possible, we have simulated a specific case of a sharpening cone for which an analytical solution exists. The results are in excellent agreement and the electric field computed with FEM and using analytical expression differ by less than $2 \%$.

\section{Equilibrium between surface tension and electrical stress}

The equilibrium between surface tension and electrical stress at the apex of the forming liquid cone can be expressed as $^{7}$

$$
\frac{2 \gamma}{r_{a}}=\frac{1}{2} \epsilon_{0} E_{a_{0}}^{2},
$$

where $\gamma$ is the surface tension, $\epsilon_{0}$ is the permittivity of free space, and $E_{a_{0}}$ is the equilibrium electrical field for a radius of curvature $r_{a}$ at the apex of the deforming liquid. The vector signs have been removed as the problem is considered to be symmetrical and the electric field lies on the axis of symmetry.
The potential difference $V_{0}$ between the emitter (capillary combined with liquid surfaces) and the extractor electrode to obtain a radius of curvature $r_{a}$ at the apex of the liquid is given by

$$
V_{0}=\frac{1}{\kappa} E_{a_{0}}=\frac{1}{\kappa} \sqrt{\frac{4 \gamma}{\epsilon_{0} r_{a}}} .
$$

In the case of a spray in ionic mode a critical electric field on the surface of the liquid needs to be reached before ions start to evaporate and are accelerated toward the extractor electrode. The critical field depends on the liquids properties, but ion evaporation is assumed to start for fields larger than 1 $\mathrm{V} / \mathrm{nm} .{ }^{17}$ To determine the critical voltages $V_{c}$, we can reuse the relationships between potential $V_{\xi}$ and electric field $E_{a_{\xi}}$ computed using FEM for various apex radii $r_{a}$. The critical voltage on the emitter necessary to initiate ion evaporation at the apex of the liquid surface is simply given by

$$
V_{c}=\frac{1}{\kappa} E_{c},
$$

where $E_{c}$ is the critical field necessary to initiate ion emission. The intersection between the critical voltage versus $r_{a}$ and the equilibrium voltage versus $r_{a}, \quad V_{\mathrm{oc}}=V_{c}\left(r_{a_{\mathrm{oc}}}\right)$ $=V_{0}\left(r_{a_{\mathrm{oc}}}\right)$, allows the determination of the onset voltage $V_{\mathrm{oc}}$ for ES operating in ionic mode. As will be shown in Sec. III C this result is valid for cone half-angles smaller than or equal to $49.3^{\circ}$. For larger angles, going from large to small apex radii, the equilibrium voltage curve reaches a maximum before decreasing again. Possible implications of this behavior will be discussed later (Sec. III C).

\section{RESULTS AND DISCUSSION}

\section{A. Microfabricated emitters}

The method was first applied to our microfabricated emitters. Their geometry and scanning electron microscopy (SEM) photographs are shown in Fig. 2. The FEM to determine the relationship between potential and electric field was created in ANSYS, following the procedures given in Sec. II B. Figure 6 shows the computed equilibrium voltages for a $20 \mu \mathrm{m}$ inner diameter capillary placed $40 \mu \mathrm{m}$ below an extractor electrode with a $120 \mu \mathrm{m}$ hole. Each point corresponds to a solution of Eq. (11), giving an equilibrium voltage for a given apex radius. Although this is the reverse of another possible approach (which would be to set a voltage, then compute the radius of curvature), the final plot provides the same information: neglecting ion emission, the radius of curvature can be determined for any applied voltage.

With decreasing apex radii $\left(r_{a}<1 \mu \mathrm{m}\right)$, the equilibrium voltage curve becomes flat and the electric field starts behaving inversely proportional to the square root of the apex radius, $1 / \sqrt{r_{a}}$, leading to the constant equilibrium voltage observed (Fig. 7). For radii above $1 \mu \mathrm{m}$, the electric field is distorted by the structure surrounding the emitters.

What happens to the shape of the liquid if the voltage is gently increased from $0 \mathrm{~V}$ up to the onset voltage? Based on the observations made during spray experiments (such as the single capillary illustrated in Fig. 3) the tip apex radius de- 


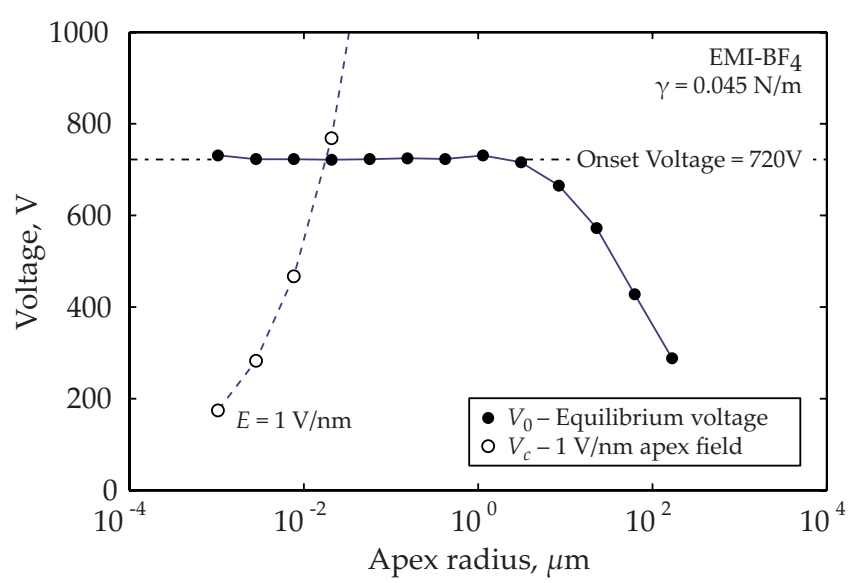

FIG. 6. (Color online) Plot of equilibrium voltage vs apex radius for an emitter with an extractor electrode that is $40 \mu \mathrm{m}$ above the capillary tip and with an extractor diameter of $120 \mu \mathrm{m}$ computed using our method (lines only added for readability). The dashed curve represents an apex field of 1 $\mathrm{V} / \mathrm{nm}$. In this case the onset value is the plateau voltage of $720 \mathrm{~V}$.

creases, and once the onset voltage is reached, the surface snaps over into a conelike structure. While remaining below the onset voltage and assuming a quasistatic change in the shape of the liquid, charge relaxation is not an issue and thus the surface remains equipotential. Once the onset voltage is reached, in Fig. 6 this occurs for an apex radius below $1 \mu \mathrm{m}$, the liquid surface sharpens and a spray is initiated.

At this point it is necessary to make a distinction between droplet and ionic modes of operation. As discussed in Sec. II C, a critical voltage exists (for ionic sprays) at which the electric field at the apex is large enough to initiate ion emission. In Fig. 6 this critical voltage has been computed using Eq. (13), assuming a field strength of $E_{c}=1 \mathrm{~V} / \mathrm{nm}$. The equilibrium and critical voltage plots, $V_{0}$ and $V_{c}$, intersect at a voltage of $720 \mathrm{~V}$, the onset voltage, and an apex radius of about $20 \mathrm{~nm}$. Note that because the equilibrium voltage versus apex radius curve exhibits a large flat region, the same onset voltage would be found for a critical field for emission of 2 or $0.5 \mathrm{~V} / \mathrm{nm}$.

For SE operating in droplet mode, after the onset voltage is reached, the notion of the critical field does not apply. In this case we assume that, above the onset voltage, the deformation into a conelike structure with a jet occurs faster than

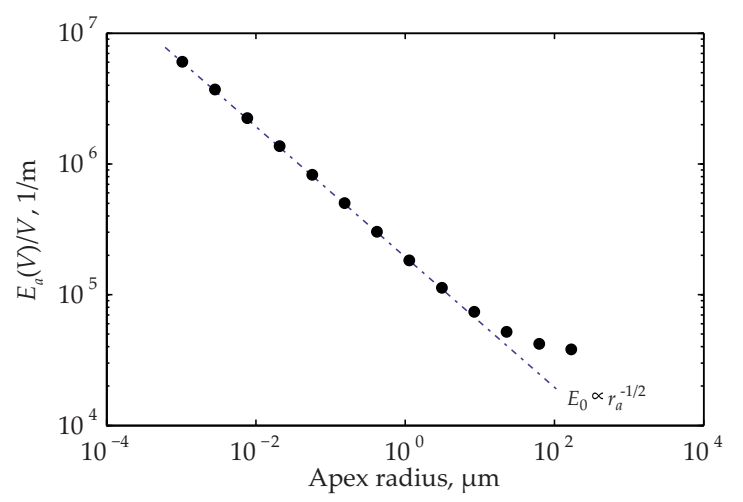

FIG. 7. (Color online) $E_{a}(V) / V$ electric field computed for the investigated MEMS geometry. The relationship $E_{a} \propto r_{a}^{-1 / 2}$ is clearly visible for apex radii of curvature less than $1 \mu \mathrm{m}$.

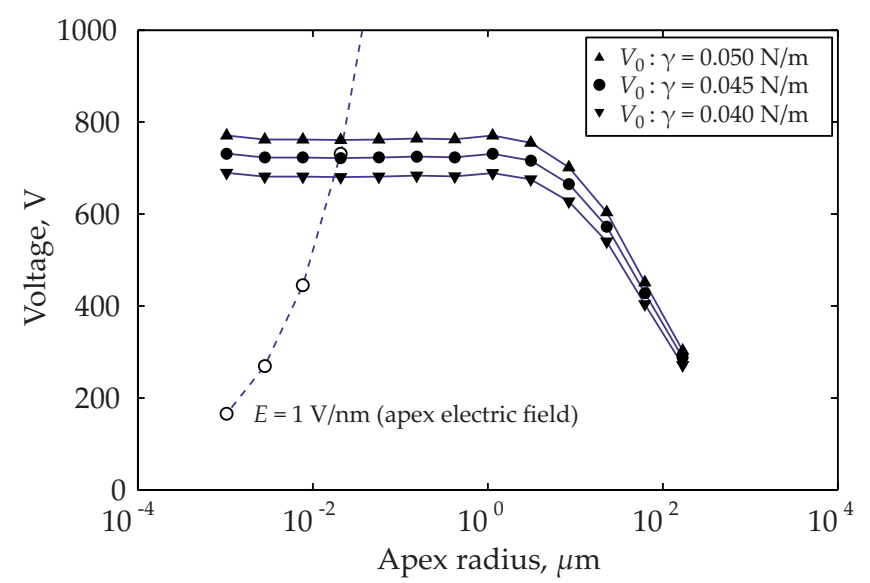

FIG. 8. (Color online) Sensitivity of the results to a variation in the surface tension. The same geometry as in Fig. 6 was used. The onset voltage dependence on the surface tension can be read from the graph. Here the onset voltage occurs at the plateau in the equilibrium voltage (lines only added for readability).

charge relaxation. The assumption of an equipotential surface is therefore no longer valid and the surface shape selected in Sec. II A no longer reproduces the behavior of the liquid. This snap-over behavior could also explain why for liquids with low conductivities, such as EMI- $f_{2} \mathrm{~N}(0.88$ $\mathrm{S} / \mathrm{m}$ ), purely ionic modes have been observed when sprayed using a needle (high fluid impedance, limited flow rate) and droplet mode when sprayed using a capillary. ${ }^{18-20}$ In the first case the surface remains at equipotential during snap-over, which occurs more slowely than charge relaxation, while in the second case the surface cannot be assumed to be an equipotential due to the liquid's high flow rate.

The method predicts the change in the onset voltage if design parameters are changed or, as shown in Fig. 8, the surface tension is varied. These results are remarkably close to the onset voltages observed in our experiments. Table I compares experimental data with the method presented in this paper and a well-established analytical model. Experimental data were acquired under vacuum conditions with the nozzles facing downward. Filling of the capillaries occurred through capillarity without any active pumping mechanism. ${ }^{3}$

The analytical model of Table I assumes a hyperboloid surface facing a planar counterelectrode, ${ }^{21}$

TABLE I. Comparison between experimental, simulation, and analytical results for our microfabricated ES arrays. The simulation results have been obtained with the method presented in this paper, while the analytical ones using the hyperboloid surface algorithm.

\begin{tabular}{ccccc}
\hline \hline \multirow{2}{*}{$\begin{array}{c}\text { Source-extractor } \\
\text { distance }^{\mathrm{a}}(\mu \mathrm{m})\end{array}$} & $\begin{array}{c}\text { Extractor } \\
\text { diameter }(\mu \mathrm{m})\end{array}$ & \multicolumn{3}{c}{ Onset voltage (V) } \\
Expt. $^{\mathrm{b}}$ & Simul. $^{\mathrm{c}}$ & Analyt. $^{\mathrm{d}}$ \\
\hline 5 & 90 & $550-570$ & 530 & 171 \\
25 & 140 & $670-690$ & 700 & 472 \\
40 & 120 & 700 & 720 & 582 \\
40 & 140 & $700-720$ & 755 & 582 \\
\hline \hline
\end{tabular}

${ }^{\mathrm{a} C a p i l l a r y}$ height of $70 \mu \mathrm{m}$ and capillary diameter of $20 \mu \mathrm{m}$.

${ }^{\mathrm{b}}$ Range of experimentally measured onset voltages at room temperature with

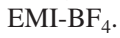

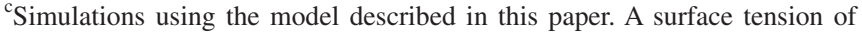
$0.045 \mathrm{~N} / \mathrm{m}$ was used for the simulation.

${ }^{\mathrm{d}}$ Analytical model assuming hyperboloid emitter surface (from Ref. 21). 


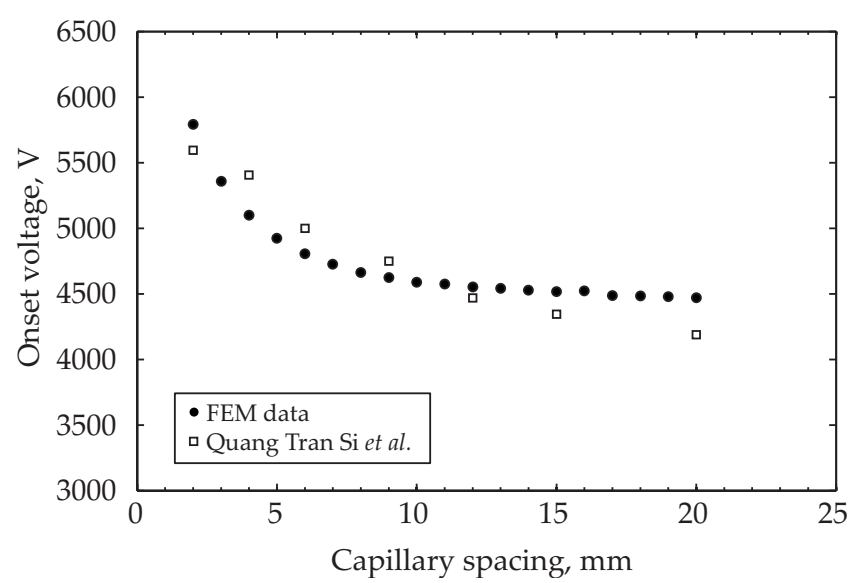

FIG. 9. Comparison between results obtained with our method and measurements from Quang Tran Si et al. (Ref. 10).

$$
V_{0}=\sqrt{\frac{\gamma R_{c}}{\epsilon_{0}}} \frac{\ln \left[\frac{R_{c}+2 d+2 \sqrt{d\left(d+R_{c}\right)}}{R_{c}}\right]}{\sqrt{1+\frac{R_{c}}{d}}},
$$

where $R_{c}$ is the tip radius of a needle and $d$ is the distance between the tip of the hyperboloid surface and the extractor electrode. For a capillary an equivalent radius needs to be selected; here $R_{c}$ was set to the capillary radius, as is generally done.

\section{B. Comparison of the model with other experimental results}

To further validate the method, the onset voltage was determined for the geometry of emitters reported by Quang Tran Si et al. ${ }^{10}$ The investigated configuration is a linear array of five stainless steel capillaries, each with an inner diameter of $0.5 \mathrm{~mm}$ and an outer diameter of $1.5 \mathrm{~mm}$. The extractor electrode is placed $7 \mathrm{~mm}$ above the flat tip of the capillaries. In their experimental setup the two external emitters were not spraying to reduce edge effects. Acetone, with surface tension of $0.0237 \mathrm{~N} / \mathrm{m}$, was fed to the capillaries by means of a syringe pump at a flow rate of $20 \mu \mathrm{l} / \mathrm{min}$. The operating voltage for the spray was defined for a fully stabilized cone on the central capillary.

For our FEM a linear array of an infinite number of emitters was chosen by modeling a single emitter and by placing cyclical constraints at the boundary of the model. Again the meshing turned out to be critical, in particular as in this case the emitter geometry is not axisymmetric and the whole capillary needs to be modeled.

The results of the model for the onset voltage are shown in Fig. 9 together with experimental data of Quang Tran Si et $a l .{ }^{10}$ As should be expected, the onset voltage increases as the capillary distance decreases. Again the correlation of results is excellent, although the liquid was pumped and it could be argued that the onset voltage could be different for nonforced flows. The difference between the two curves can be explained by the limited number of emitters in the experiment and the edge effects, tending to deform the liquids from an axisymmetric geometry.

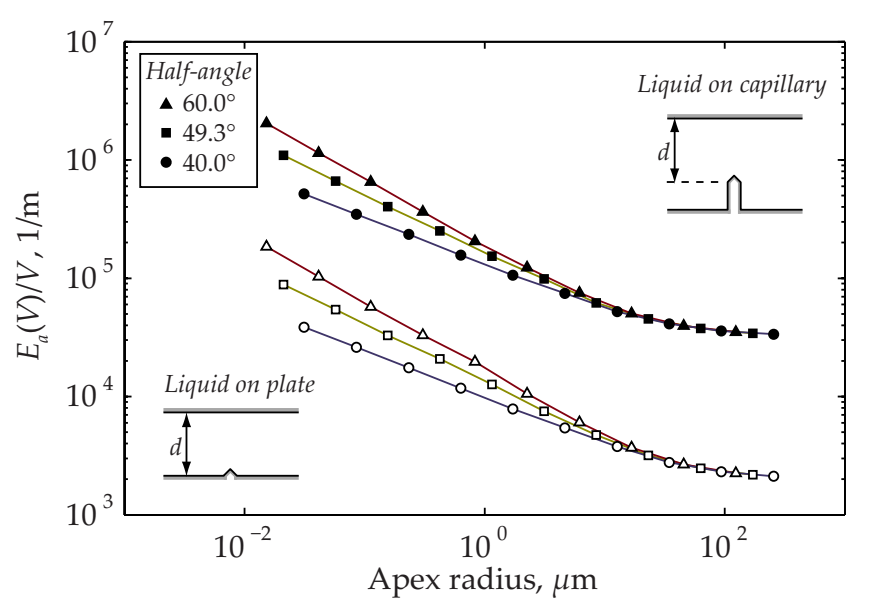

FIG. 10. (Color online) $E_{a}(V) / V$ computation for two emitter geometries and three different cone half-angles. Upper curves: liquid on the tip of a capillary; lower curves: liquid on a flat surface.

\section{Further discussion}

In the previous paragraph (Secs. III A and III B) the computed equilibrium apex electric field scaled as $1 / \sqrt{r_{a}}$ with a cone half-angle of $49.3^{\circ}$. This behavior is valid as long as the apex-extractor distance is much larger than the apex radius of curvature, i.e., $d \gg r_{a}$. To illustrate this, Fig. 10 shows the evolution of $E_{a}(V) / V$ as a function of the apex radius. Two configurations were chosen: a liquid deforming on the tip of a needle and one on a flat surface. For both geometries the extractor electrode was placed at $500 \mu \mathrm{m}$ from the base of the cone and the radius at the base of the cone was $10 \mu \mathrm{m}$.

For both geometries the shapes of the curves are identical except for a shift in the field strength. The three different slopes were obtained by varying the cone half-angle. This is interesting because research by Yarin et al. ${ }^{22}$ identified cone half-angles around $33.5^{\circ}$ for liquids having nonrelaxing elastic forces. The existence of cone half-angles larger than $49.3^{\circ}$ have been observed by Driesel et al. ${ }^{11}$ for liquid metal ion sources. ${ }^{11}$ For certain metals, such as gallium, they have measured an angle of almost $54^{\circ}$ for very low emission currents. In analogy with the behavior of the field near a sharp point or conical hole, ${ }^{23}$ we can write

$$
E \propto r_{a}^{\nu-1}
$$

where $\nu$ is the solution of the equation

$$
P_{\nu}[\cos (\pi-\beta)]=0
$$

with $P_{\nu}$ being the Legendre polynomial of the order of $\nu$.

Table II summarizes the values $\nu-1$ extracted from Fig. 10 and compares them to the analytical results obtained solving Eq. (16). The results show a very good agreement between the analytical values and the computed ones. Therefore, for an extractor electrode placed far away from the liquid, it can be concluded that once a certain apex radius is reached, the behavior of the electric field will only be influenced by the cone half-angle and not by the surrounding geometry. The consequence of this observation is that for a cone half-angle of $49.3^{\circ}$ the field will behave as $1 / \sqrt{r_{a}}$ and in 
TABLE II. Comparison of the slopes determined solving Eq. (16) and extracted from the FEM.

\begin{tabular}{lcccc}
\hline \hline Geometry $^{\mathrm{a}}$ & $\beta^{\mathrm{b}}$ & $\nu^{\mathrm{c}}$ & $\nu-1$ & FEM $^{\mathrm{d}}$ \\
\hline $\mathrm{C}$ & 40.0 & 0.422 & -0.578 & -0.565 \\
$\mathrm{~S}$ & 40.0 & 0.422 & -0.578 & -0.567 \\
$\mathrm{C}$ & 49.3 & 0.500 & -0.500 & -0.482 \\
$\mathrm{~S}$ & 49.3 & 0.500 & -0.500 & -0.491 \\
$\mathrm{C}$ & 60.0 & 0.602 & -0.398 & -0.390 \\
$\mathrm{~S}$ & 60.4 & 0.602 & -0.398 & -0.394 \\
\hline
\end{tabular}

${ }^{a}$ Geometry: (C) liquid on capillary; (S) liquid on plate.

${ }^{\mathrm{b}}$ Cone half-angle.

${ }^{\mathrm{c}}$ Solution of Eq. (16)

${ }^{\mathrm{d}}$ Slope $\nu-1$ computed from FEM.

order to compute the onset voltage one single FEM model having a $w \gg 1$ [i.e., $\left.r_{a} \ll p_{x} \tan (\beta)\right]$ is sufficient. This also explains why the commonly used hyperbolic or parabolic surface model provides satisfactory results for slender needles placed far away from the extractor electrode.

The determination of the onset voltage is more complex for tip geometries with cone half-angles smaller or larger than the Taylor cone angle. These geometries require the computation of the entire equilibrium voltage curve. Figure 11 illustrates the equilibrium voltage behavior for the capillary geometry (10 $\mu \mathrm{m}$ radius, $500 \mu \mathrm{m}$ extractor distance). For the Taylor cone angle, $49.3^{\circ}$, we obtain a flat onset voltage curve for small apex radii, while for an angle of $40^{\circ}$ the equilibrium voltage increases steadily as $r_{a}$ decreases. For a cone half-angle of $60^{\circ}$ there is a maximum equilibrium voltage for a finite $r_{a}$ value, i.e., the curve is nonmonotonic. The

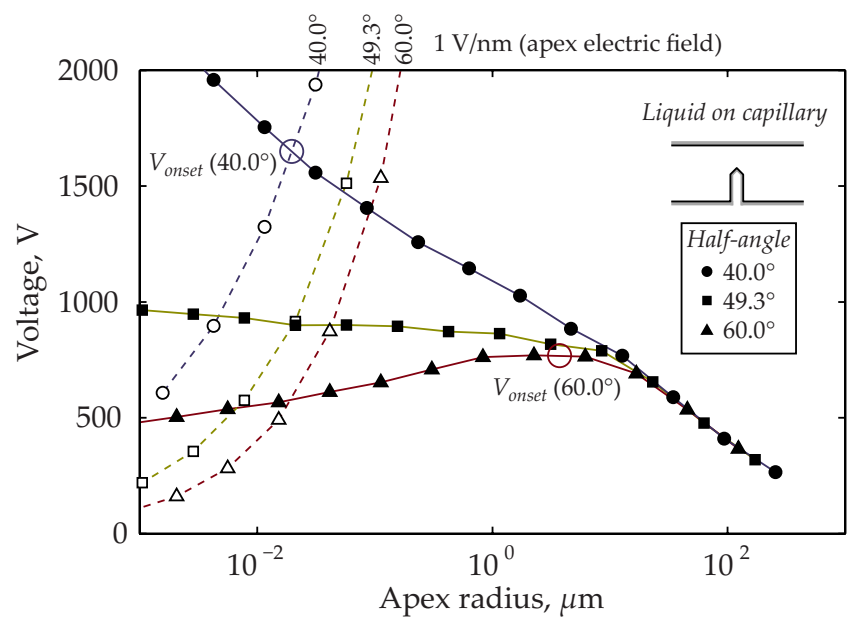

FIG. 11. (Color online) Equilibrium voltage curves on the tip of a capillary for three different cone half-angles. The solid lines represent the equilibrium voltage. The dashed lines represent, for each individual cone half-angle, the $1 \mathrm{~V} / \mathrm{nm}$ field at the apex. For cone angles of $49.3^{\circ}$ (Taylor cone angle), the onset voltage is equal to the value of the plateau in the equilibrium voltage. For cone angles below $49.3^{\circ}$ (top solid curve), the onset voltage can only be computed if a "threshold field" for emission is known. As example the intersection with the $1 \mathrm{~V} / \mathrm{nm}$ apex field is marked (upper left circle). For angles below 49.3 (bottom solid curve) there is a maximum in the equilibrium voltage vs $r_{a}$ curve. In that case the onset voltage is the maximum in the equilibrium voltage curve (lower right circle) since as the voltage is ramped up from zero, the radius of curvature will decrease continuously until the equilibrium voltage maximum is reached, at which point the liquid snaps over to a cone- or a cusplike shape and ion/droplet emission begins. three dashed curves in Fig. 11 correspond to a tip field of 1 $\mathrm{V} / \mathrm{nm}$ and have been computed for each cone half-angle using Eq. (13).

These results are of particular interest for ES sources operating in ionic mode and could explain as to why a difference is observed between onset and extinction voltage. For a $60^{\circ}$ cone half-angle the equilibrium curve crosses a maximum which would, in this example, correspond to the onset voltage of approximately $770 \mathrm{~V}$ (marked with circle in Fig. 11). If we now assume that the field self-enhances at the liquids apex, the radius will also decrease up to the point where the critical field for ion emission is reached (in Fig. 11 this is the intersection with the red, dashed curve representing a field of $1 \mathrm{~V} / \mathrm{nm}$ ). At this point the equilibrium voltage to sustain the critical field $(\approx 580 \mathrm{~V})$ is below the maximum voltage $(\approx 770 \mathrm{~V})$ and could therefore explain a difference between extinction and onset voltage. Since this study only considers the quasistatic case, no direct information on the dynamic behavior of the liquid can be gained after a snapover.

For a cone half-angle of $30^{\circ}$ a constant increase in the equilibrium voltage for a decreasing apex radius is computed. In Fig. 11 the $1 \mathrm{~V} / \mathrm{nm}$ tip field curve crosses at $1650 \mathrm{~V}$. In this case as the voltage is increased no snap-over happens and the knowledge of a threshold field for emission is necessary to determine the onset voltage. For angles below $49.3^{\circ}$ the method is therefore only valid for sprays in ionic mode where a "critical field" for ion emission is known.

\section{CONCLUSIONS}

This paper reports on a method to compute the onset voltage for ion or droplet emission from a single or from an array of ES emitters with complex geometries. The model takes as input the geometry of the capillary and of the extraction electrode as well as the surface tension of the sprayed liquid. The key assumption in this model concerns the shape of the liquid surface that deforms (decreasing radius of curvature at the apex) under the influence of the increasing electric field. Based on the experimental data, we chose a family of conic sections to represent the liquid surface.

The equilibrium voltage, representing the balance between surface tension and electric stress, is computed by means of a FEM for a number of radii of curvature at the liquid apex. This provides a plot of radius of curvature $r_{a}$ versus applied voltage $V_{\text {capillary, }}$ ignoring space charge effects, from which the onset voltage might be determined.

Noting the similarity in the behavior of the scaling of the equilibrium electric field versus radius of curvature for liquid surfaces deforming within the asymptotes of the Taylor cone half-angle $\left(49.3^{\circ}\right)$ leads to a further simplification of the method. For geometries where the extractor electrodes distance from the capillary tip $d$, is significantly larger than the apex radius $\left(d / r_{a} \gg 1\right)$, the electric field scales as $1 / \sqrt{r_{a}}$ for small apex radii. This leads to a flat equilibrium voltage curve versus radius of the curvature. This has two important consequences. (1) It is not necessary to compute the full critical voltage versus $r_{a}$ plot; it suffices to compute the equi- 
librium voltage for one single apex radius as long as this apex radius of curvature is sufficiently small $\left[r_{a}\right.$ $\left.\ll p_{x} \tan (\beta)\right]$. The necessary apex radius to compute for a reliable result is still several orders of magnitude larger than the one necessary to initiate particle emission. This results in a geometry which is much simpler to mesh when creating a FEM and explains also why analytical models calculating the equilibrium voltage at the tip of needles work well for high aspect ratio needles or capillaries. (2) Having a flat equilibrium voltage versus $r_{a}$ curve means that once this "plateau voltage" is reached, a snap-over to smaller $r_{a}$ can be expected. This has several implications for our method. On one hand, it allows predicting the onset voltage of sprays operating in droplet mode without any knowledge of the dynamic behavior of the liquid surface by assuming that once the plateau voltage is reached the liquid surface snaps over into a droplet emission. On the other hand, as discussed in Sec. III A, for cone jets operating in ionic mode the onset voltage is insensitive to the exact value of the critical field for emission. This is important because the critical field for emission is often not well known, yet this method still allows very precise predictions of the onset voltage with no free parameters.

For other cone half-angles the electric field curves have been found to behave in a similar way as a field near a sharp point or conical hole. This finding could simplify the determination of the onset voltage for other liquid geometries.

The method has been applied to a single and arrays of capillaries. The onset voltages calculated for a micromachined and a macroscopic systems are in excellent agreement with experimental data. For both cases, the complex geometries preclude determining the onset voltage by analytical means. This onset voltage determination technique is a powerful design tool for ES emitters, allowing the onset voltage to be rapidly computed for different geometries and for different liquids, thus enabling, for instance, high uniformity to be obtained, or geometries to be tuned for specific liquids.

\section{ACKNOWLEDGMENTS}

The authors are indebted to Professor John P. W. Stark (Queen Mary, University of London) for many ideas and several important insights on electrosprays.

${ }^{1}$ J. Xiong, Z. Zhou, D. Sun, and X. Ye, Sens. Actuators, A 117, 168 (2005).

${ }^{2}$ W. Deng, J. F. Klemic, X. Li, M. A. Reed, and A. Gomez, J. Aerosol Sci. 37, 696 (2006)

${ }^{3}$ R. Krpoun, M. Räber, and H. R. Shea, Proceedings of the 21st IEEE International Conference on Micro Electro Mechanical Systems (IEEE, New York, 2008), pp. 964-967.

${ }^{4}$ R. Bocanegra, D. Galan, M. Marquez, I. G. Loscertales, and A. Barrero, J. Aerosol Sci. 36, 1387 (2005).

${ }^{5}$ L. F. Velásquez-García, A. I. Akinwande, and M. Martínez-Sánchez, J. Microelectromech. Syst. 15, 1272 (2006).

${ }^{6}$ B. Gassend, L. F. Velásquez-García, A. I. Akinwande, and M. MartínezSánchez, Proceedings of the 21st IEEE International Conference on Micro Electro Mechanical Systems (IEEE, New York, 2008), pp. 976-979.

${ }^{7}$ G. L. R. Mair, Nucl. Instrum. Methods 172, 567 (1980).

${ }^{8}$ P. Prewett and G. Mair, Focused Ion Beams from Liquid Metal Ion Sources, Electronic and Electrical Engineering Research Studies: Microengineering Series (Research Studies, 1991), Chap. 3, p. 47.

${ }^{9}$ J. D. Regele, M. J. Papac, M. J. A. Rickard, and D. Dunn-Rankin, J. Aerosol Sci. 33, 1471 (2002).

${ }^{10}$ B. Quang Tran Si, D. Byun, and S. Lee, J. Aerosol Sci. 38, 924 (2007).

${ }^{11}$ W. Driesel, C. Dietzsch, and R. Mühle, J. Vac. Sci. Technol. B 14, 3367 (1996).

${ }^{12}$ G. I. Taylor, Proc. R. Soc. London, Ser. A 280, 383 (1964).

${ }^{13}$ I. Marginean, L. Parvin, L. Heffernan, and A. Vertes, Anal. Chem. 76, 4202 (2004).

${ }^{14}$ M. S. Alexander, M. D. Paine, and J. P. W. Stark, Anal. Chem. 78, 2658 (2006).

${ }^{15}$ I. D. Faux and M. J. Pratt, Computational Geometry for Design and Manufacture (Halsted Press, Wiley, New York, 1979), Chap. 5, p. 132.

${ }^{16}$ L. D. Landau, E. M. Lifshitz, and L. P. Pitaevskiř, Electrodynamics of Continuous Media, Course of Theoretical Physics, 2nd ed. (Pergamon, New York, 1984), Vol. 8.

${ }^{17}$ M. Gamero-Castaño and J. Fernández de la Mora, J. Chem. Phys. 113, 815 (2000).

${ }^{18}$ D. Garoz, C. Bueno, C. Larriba, S. Castro, I. Romero-Sanz, J. De La Mora, Y. Yoshida, and G. Saito, J. Appl. Phys. 102, 064913 (2007).

${ }^{19}$ M. Gamero-Castaño, J. Fluid Mech. 604, 339 (2008).

${ }^{20}$ S. Castro, C. Larriba, J. De La Mora, P. Lozano, S. Sümer, Y. Yoshida, and G. Saito, J. Appl. Phys. 102, 094310 (2007).

${ }^{21}$ M. Martínez-Sánchez, MIT OpenCourseWare, 2004 (http://ocw.mit.edu/).

${ }^{22}$ A. L. Yarin, S. Koombhongse, and D. H. Reneker, J. Appl. Phys. 90, 4836 (2001).

${ }^{23}$ J. D. Jackson, Classical Electrodynamics, 3rd ed. (Wiley, New York, 1999). 